\title{
Deep Arteriovenous Malformations in the Basal Ganglia, Thalamus, and Insula: Microsurgical Management, Techniques, and Results
}

\author{
Matthew B. Potts, MD1, William L. Young, MD ${ }^{1,2,3}$, and Michael T. Lawton, MD ${ }^{1,3}$ for the \\ UCSF Brain AVM Study Project \\ 1Department of Neurological Surgery, University of California, San Francisco \\ ${ }^{2}$ Department of Anesthesia and Perioperative Care, University of California, San Francisco \\ ${ }^{3}$ Center for Cerebrovascular Research, University of California, San Francisco
}

\begin{abstract}
Background-Arteriovenous malformations (AVMs) in the basal ganglia, thalamus, and insula are considered inoperable given their depth, eloquence, and limited surgical exposure. While many neurosurgeons opt for radiosurgery or observation, others have challenged the belief that deep AVMs are inoperable. Further discussion of patient selection, technique, and multimodality management is needed.
\end{abstract}

Objective-To describe and discuss the technical considerations of microsurgical resection for deep-seated AVMs.

Methods-Patients with deep AVMs who underwent surgery during a 14-year period were reviewed using a prospective AVM registry.

Results-Microsurgery was performed in 48 patients with AVMs in the basal ganglia $(n=10)$, thalamus ( $\mathrm{n}=13)$, or insula $(\mathrm{n}=25)$. The most common Spetzler-Martin grade was III- $(68 \%)$. Surgical approaches included transsylvian (67\%), transcallosal (19\%), and transcortical (15\%). Complete resection was achieved in 34 patients $(71 \%)$, and patients with incomplete resection were treated with radiosurgery. Forty-five patients $(94 \%)$ were improved or unchanged (mean follow-up 1.6 years).

Conclusion-This experience advances the notion that select deep AVMs may be operable lesions. Patients were highly selected for small size, hemorrhagic presentation, young age, and compactness - factors embodied in the Spetzler-Martin and Supplementary grading systems. Overall, 10 different approaches were used, exploiting direct, transcortical corridors created by hemorrhage or maximizing anatomical corridors through subarachnoid spaces and ventricles that minimize brain transgression. The same cautious attitude exercised in selecting patients for

Copyright () 2013 Neurosurgery. Published by Lippincott Williams \& Wilkins.

Correspondence: Michael T. Lawton, MD, Department of Neurological Surgery, University of California, San Francisco, 505 Parnassus Ave., M780, Box 0112, San Francisco, CA 94143-0112, lawtonm@ neurosurgery.ucsf.edu.

Disclosure: The authors have no personal financial or institutional interest in any of the drugs, materials, or devices described in this article. 
surgery was also exercised in deciding extent of resection, opting for incomplete resection and radiosurgery more than with other AVMs to prioritize neurological outcomes.

\section{Keywords}

Arteriovenous malformation; Basal ganglia; Insula; Microsurgery; Thalamus

\section{Introduction}

Convexity arteriovenous malformations (AVM) in the frontal, temporal, parietal, and occipital lobes are approached with simple craniotomies that widely expose the nidus for safe, perpendicular dissection of margins. AVMs in the basal ganglia, thalamus, and insula, while still supratentorial, are deeper and require more elaborate surgical approaches. Such surgical approaches provide limited exposure and dissection of margins is more tangential, making them more challenging. In addition, proximity to critical structures like the internal capsule makes surgical resection of deep AVMs more risky than convexity AVMs. Consequently, many neurosurgeons immediately opt for radiosurgery or observation. However, deep location and deep venous drainage contribute to an aggressive natural history, with annual hemorrhage rates reported between $10 \%$ and $34 \%,{ }^{1,2}$ associated hemiparesis rates as high as $85 \%,{ }^{1}$ and mortality rates up to $62.5 \%{ }^{3}$ Deep AVMs also have increased risks associated with radiosurgical therapy because the basal ganglia and thalamus are exquisitely sensitive to radiation side effects and hemorrhage during the latency period. ${ }^{4-6}$ Finally, radiosurgical obliteration rates with deep AVMs are lower than in other locations. ${ }^{5}$ Experienced AVM surgeons have challenged the belief that deep AVMs are inoperable, demonstrating in literature review a complete resection rate $(91 \%)$ that exceeds the radiosurgical obliteration rate $(69 \%)$ with lower mortality $(2.4 \%)$ than that resulting from latency-period hemorrhage (5.3\%). ${ }^{7}$ This re-evaluation of surgery for deep AVMs is appropriate, but further discussion of patient selection, surgical technique, and multimodality management strategy is needed. We present our surgical experience with 48 patients with basal ganglia, thalamic, and insular AVMs to advance this notion that deep AVMs in these regions can be operated with acceptable morbidity rates and good cure rates, making microsurgery preferable to radiosurgery or observation in carefully selected patients.

\section{Patients and Methods}

\section{Data Collection}

This study was approved by the Institutional Review Board of the University of California, San Francisco, and conducted in compliance with Health Insurance Portability and Accountability Act regulations. The prospective registry of the UCSF Brain Arteriovenous Malformation Study Project was searched to identify patients with insular, basal ganglia, and thalamic AVMs who were treated with surgical resection between 1997 and 2011. This database, medical records, pre- and postoperative radiographic studies, intraoperative photographs, and clinical follow-up evaluations were retrospectively reviewed. 


\section{Patient Selection}

Patients with AVMs of the basal ganglia, thalamus, and insula are reviewed by our institution's multidisciplinary cerebrovascular team, which includes neurosurgeons, neurologists, interventional neuroradiologists, and radiation oncologists. In general, surgical preference is given to small AVMs with compact niduses, superficial venous drainage, and hemorrhagic presentation. Patients with poorer functional status at presentation are also often selected for surgical resection. In addition, surgery is considered in patients with prior radiosurgical treatment who have hemorrhaged or whose AVM volume sufficiently decreased to make surgery safer.

\section{Microsurgical Approaches}

Three main categories of approaches were used to access these deep AVMs: transsylvian, transcallosal, and transcortical (Table 1, Figure 1). Transsylvian approaches begin with a wide splitting of the Sylvian fissure to access the opercular surfaces of the frontal and temporal lobes and insula. Once the insula is exposed, the AVM is accessed directly if it lies on the insular surface or transinsularly if it lies below insular cortex. We previously defined the anterior and posterior transinsular approaches as two distinct variations of the transsylvian approach. ${ }^{1,2,8}$ The anterior approach uncovers the sphenoidal (M1) and insular (M2) branches of the (MCA) to expose the limen insulae and short gyri, whereas the posterior approach is directed at the opercular (M3) MCA branches and exposes the circular sulcus and long gyri of the insula. The anterior approach requires a standard pterional craniotomy while the posterior approach requires more posterior exposure of the distal Sylvian fissure with a frontotemporoparietal craniotomy. A third variation of the transsylvian approach is the supracarotid-infrafrontal approach, ${ }^{1,9}$ which exploits the supracarotid triangle between the A1 segment of the anterior cerebral artery and the M1 segment of the MCA to pass medial and inferior to the insula, through the medial orbital gyrus of the frontal lobe. The trajectory of this approach is directed superiorly, and therefore requires an orbitozygomatic craniotomy to increase the upward viewing angle.

The transcallosal approaches expose the midline, dissect the interhemispheric fissure, traverse the corpus callosum, and extend transventricularly to reach targets medially through the ependyma of the lateral ventricle. ${ }^{3,10,11}$ These approaches do not transgress any cortical tissue, only a portion of corpus callosum. Variations include anterior, posterior, ipsilateral, contralateral, and transchoroidal fissure. The anterior-ipsilateral transcallosal approach offers a direct view of the floor of the lateral ventricle and superior thalamus, whereas the anterior-contralateral transcallosal creates a steeper viewing angle across to the lateral wall of the ventricle and caudate nucleus. The transcallosal-transchoroidal approach deepens the exposure by opening the teniafornicea of the choroid plexus, following the thalamostriate and internal cerebral veins into the roof of the third ventricle, and widening the foramen of Monroe to create an entrée into the third ventricle. This approach accesses the lateral wall of third ventricle and medial thalamus. The posterior transcallosal approach transgresses the splenium for a more posterior view of the superior thalamus, as well as arterial feeders and draining veins around the Galenic complex that are inaccessible from anterior transcallosal approaches. All of the transcallosal variations involve a craniotomy that crosses the midline to expose the sagittal sinus, with the anterior approaches using a bifrontal craniotomy and 
the posterior approach using a torcular craniotomy that exposes the junction of superior sagittal and transverse sinuses. Gravity was used to retract the dependent hemisphere and open the interhemispheric fissure in all transcallosal variations. The anterior approaches use a supine position with the head turned to align the midline horizontally, and the posterior approach uses a lateral position also with the head turned to align the midline horizontally. Again, gravity retraction enables retractorless exposure of these routes.

Transcortical approaches are used when hematoma or encephalomalacia from prior hemorrhage creates a nonanatomical corridor to the AVM that would otherwise not exist. ${ }^{4-6,12}$ These cavities directly access deep AVMs with minimal additional transgression through the cortex. Transcortical routes can also be used in the absence of hematoma or encephalomalacia, but cortical transgression can produce new neurological deficits for which the neurosurgeon is responsible. Transcortical routes are selected in regions known to be safe, such as the middle and inferior temporal gyri and superior parietal lobule. ${ }^{5,13}$ The corridors are long and without anatomical landmarks, necessitating neuronavigation and some brain retraction.

\section{Outcome Evaluation}

Neurological outcome was assessed using the Rankin Scale (RS). Neurologic assessments were performed by a neurologist, neurosurgeon, or associated nurse clinician. The pretreatment RS was obtained from routine pre-treatment clinic visits or admission physicals. Follow-up information was obtained during routine post-treatment clinic visits, subsequent hospital admissions, or telephone interviews. Good outcomes were defined as a final RS score of 1 to 2 while poor outcomes were defined as a final RS greater than 2. Functional improvement was defined as a decrease in the RS score from the preoperative exam to the final follow-up exam. Deterioration was likewise defined as an increase in the RS score. Angiographic outcomes after surgery were defined as either complete or incomplete AVM resection.

\section{Statistical Analysis}

Statistical analysis was performed using JMP 10.0 (SAS, North Carolina, USA). Frequency distributions and summary statistics were calculated for all pre- and postoperative characteristics. For categorical variables (i.e., Spetzler-Martin grade, etc.), cross-tabulations were generated and $\chi^{2}$ tests were used to compare distributions among the three location groups (insula, basal ganglia, and thalamus). Continuous variables (i.e., age) were compared with a t-test. Univariate and multivariate analyses of preoperative factors associated with complete resection were performed with a stepwise logistic regression model. A mixeddirection stepwise regression analysis with p-value thresholds of 0.2 to enter and leave the model was used to identify variables to be included in the final logistic regression model. Statistical significance was defined as $p<0.05$. 


\section{Results}

\section{Patients}

During a 14-year period from August 1997 to August 2011, 514 patients with brain AVMs were treated surgically by the senior author. Of these, 48 (9\%) had basal ganglia, thalamic, or insular AVMs. The mean patient age was 31 years, with 21 women (44\%) and 27 men (56\%, Table 2). The most common presentation was hemorrhage (77\%), followed by headache (10\%), seizures ( $8 \%)$, focal sensory deficit (2\%), and incidental diagnosis after head trauma (2\%).

Ten AVMs (21\%) were located in the basal ganglia, 13 (27\%) in the thalamus, and $25(52 \%)$ in the insula. Thirty-four AVMs (70\%) were Spetzler-Martin grade III, and $68 \%$ of these were small (modified III-, Table 2). Thalamic AVMs were significantly more likely than insular and basal ganglia AVMs to have deep drainage ( $\mathrm{p}=0.01)$. High incidences of hemorrhagic presentation (77\%) and compact nidus (88\%) resulted in low Supplementary AVM grades ( $81 \%$ grade I - III, Table 2$)$.

No patient had prior surgery for AVM resection but 7 patients underwent prior hematoma evacuation and 1 underwent clipping of an unrelated, unruptured aneurysm. Seven patients (15\%) underwent non-surgical AVM treatment prior to surgical resection, including embolization in 3 (not including preoperative embolization) and gamma knife radiosurgery in 6 ( 2 patients received both modalities). Three of the six radiosurgical patients had suffered hemorrhages during the latency period after radiosurgical treatment.

\section{Microsurgical AVM Management}

Preoperative embolization was performed in only 21 patients (44\%), reflecting the difficulty in catheterizing deep perforators that supply these AVMs, including the lenticulostriates, insular perforators, thalamoperforators, and the anterior choroidal artery (AChA). Preoperative embolization was more commonly used for thalamic and insular AVMs (46\% and 56\%, respectively) compared to basal ganglia AVMs $(10 \%, \mathrm{p}=0.045)$.

Of the three main categories of approaches used to access these deep AVMs, transsylvian approaches were the most common (67\%), followed by transcallosal (19\%) and transcortical (15\%). Table 3 summarizes the various approaches used based on AVM location. Insular AVMs were the largest subgroup of AVMs, making the transsylvian-transinsular approaches the most utilized ( 31 total patients, 15 anterior and 16 posterior). Transsylvian approaches were mainly used to access AVMs in the insula and basal ganglia. The supracarotidinfrafrontal approach, ${ }^{7,9}$ an anteroinferior variation that dissects through medial orbital gyrus rather than insula, was used with 1 basal ganglia AVM.

The transcallosal approaches were used in 9 patients (19\%) to access AVMs of the basal ganglia and thalamus. The contralateral approach was the only transcallosal approach used with basal ganglia AVMs, providing a good working angle to the caudate nucleus from the opposite side of the interhemispheric fissure. Thalamic AVMs were accessed with all variations, depending on whether the nidus was in the superior thalamus (anterior-ipsilateral 
transcallosal, 2 patients), posterior thalamus (posterior-ipsilateral transcallosal, 1 patient), or medial thalamus (transcallosal-transchoroidal, 4 patients).

Transcortical corridors were used in 7 patients, typically those with hemorrhage and either hematomas or encephalomalacia that extended to or near the cortical surface. Transcortical approaches were used with all subtypes of deep AVMs, but mainly with thalamic AVMs (5 patients).

Neuronavigation was used for 11 patients (7 patients with thalamic AVMs and 4 patients with insular AVMs). Awake language mapping was utilized in 1 patient as part of the second stage of resection of an insular AVM. Motor mapping was not used. Seven patients $(15 \%)$ required staged resections, six of which were found to have residual AVM on postoperative angiogram that was deemed resectable and one patient whose initial case was aborted in favor of returning to the OR for awake language mapping. Two of these patients with staged resections hemorrhaged in between stages and a gross total resection was achieved during the second stage.

Complete AVM resection, as measured by postoperative cerebral angiogram prior to discharge, was achieved in 34 patients (71\%, Table 4). Thalamic AVMs were less likely than insular and basal ganglia AVMs to be completely resected. Of the 14 patients with a subtotal resection, dissection of the AVM was intentionally halted in seven because of deep penetration of the nidus into the thalamus and concern for thalamic injury with further dissection (five patients). Four of these thalamic AVMs presented with hemorrhage, which we thought would help define a surgical plane intraoperatively. In addition, one patient with a thalamic AVM had failed prior radiosurgical treatment. Another thalamic AVM resection was halted due to changes in neuromonitoring. In addition, a posterior insular AVM located beneath the speech area, based on awake language mapping, could not be exposed through a posterior transsylvian approach without transgression through language cortex. In the remaining seven patients with subtotal resection, remnant AVM was found unexpectedly on postoperative angiogram, but was deemed in most cases to be small, in a location that might risk new neurological deficit with re-operation, and amenable to radiosurgical treatment. Overall, 12 of the 14 patients with subtotal resection presented with hemorrhage and 2 failed prior radiosurgical treatment. The nidus size was $<3 \mathrm{~cm}$ in 8 patients and $3-6 \mathrm{~cm}$ in 6 patients.

Of the 14 patients with a subtotal resection, 9 underwent subsequent gamma knife radiosurgery at our institution to treat the AVM remnant. Of those, 1 patient suffered a hemorrhage during the latency period 8 months after surgical resection and underwent a complete resection at that time. Five patients are currently in the latency period, and 4 have had 1-year post-radiosurgery MRIs showing 3 stable AVMs and 1 AVM that decreased in size. The remaining 3 patients have not been evaluated at our institution since their radiosurgery.

Multivariate analysis examining the individual variables within the Spetzler-Martin and Supplemental AVM scores, AVM location (basal ganglia, thalamus, or insula), and use of preoperative embolization identified AVM location $(\mathrm{p}=0.03)$ and patient age $(\mathrm{p}=0.016)$ as 
factors associated with complete AVM resection. Insular and basal ganglia location combined had an odds ratio of 2.4 compared to thalamic AVMs (95\% CI, 1.13-5.93). Older age ( $>40$ years) had an odds ratio of 3.3 compared to younger patients ( $\$ 40$ years, $95 \% \mathrm{CI}$, 1.2-15.5), suggesting perhaps that some brain atrophy improves surgical exposure of these deep AVMs.

\section{Complications and Outcomes}

Complications were recorded in 14 patients (29\%). Two patients hemorrhaged after the first of 2 surgical stages and underwent complete resection during the second stage. As described above, an additional patient suffered a hemorrhage after gamma knife radiosurgery for their residual AVM. Four patients had seizures postoperatively. One patient had a single generalized seizure postoperatively in hospital; 2 patients had seizures after tapering off anticonvulsant medications 14 and 21 months postoperatively; and the fourth patient developed seizures 2 years postoperatively. Four patients presenting with hemorrhage developed hydrocephalus requiring ventriculoperitoneal shunts. Two patients developed postoperative wound infections requiring debridement. Finally, 1 patient suffered a posterior cerebral artery (PCA) stroke during a preoperative embolization of a thalamic AVM, resulting in arm and hand weakness. After postponing surgery for 6 months, their weakness improved and the AVM was completely resected. Complications in 12 of these 14 patients $(86 \%)$ were minor, attributable to non-surgical aspects of management, or did not result in significant neurological deficits.

There was no surgical mortality in this patient series (Table 4). Three patients had new neurological deficits at time of hospital discharge that resolved completely at later follow-up (transient neurologic morbidity, 6\%). At late follow-up, 3 patients had a worsened RS score (permanent neurologic morbidity, 6\%; mean length of follow-up, 1.6 years). These included 1 patient with postoperative hemorrhage, 1 who hemorrhaged after subsequent gamma knife radiosurgery of their residual AVM, and a third who developed shunt-dependent hydrocephalus. Overall, good outcomes (RS 1-2) were observed in 39 patients (81\%) while poor outcomes ( $\mathrm{RS} \geq 3$ ) were observed in 9 patients $(19 \%$, Figure 2$)$.

\section{Discussion}

\section{Patient Selection}

Most neurosurgeons consider surgery for deep AVMs in the insula, basal ganglia, and thalamus to be risky and consequently manage them conservatively or with radiosurgery, with or without embolization. Our surgical experience with 48 such AVMs, or $9 \%$ of our total surgical cohort, demonstrates that these patients are more amenable to surgery than expected. Although our surgical series is large, these patients were highly selected for hemorrhagic presentation, young patient age, compactness, and size. Only 1 of these selection criteria - size - comes from the Spetzler-Martin grading system. Sixty-seven percent of patients had small AVMs $<3 \mathrm{~cm}$ diameter and 68\% had Spetzler-Martin grade IIIAVMs, which is the grade III subtype most favorable for surgery according to our modification of the grading system. ${ }^{14}$ Since the majority of these deep AVMs were, by 
definition, in eloquent brain (98\%) or had deep venous drainage (73\%), the Spetzler-Martin grade was less important in patient selection than it is for AVMs in other locations.

In contrast, the components of the Supplementary grading system were critically important. ${ }^{15}$ Most of the patients selected for surgery were $\$ 40$ years (69\%), had ruptured AVMs (77\%), and had a compact nidus (88\%). Therefore, the Supplementary grade is a useful guide to surgical decisions with deep AVMs and helps to achieve an appropriate index of selectivity. There were few patients in our surgical series with high supplementary scores (IV or greater) or high combined Spetzler-Martin + Supplementary scores (VI or greater).

Eight patients in this series (17\%) were managed non-operatively with other treatment modalities before undergoing surgery. In addition, 60 patients with AVMs in the basal ganglia, thalamus, and insula were managed non-operatively during the same study period and were not included in this review. These numbers demonstrate that only a small group of patients have the clinical and pathological factors necessary to overcome a cautious management attitude.

\section{Deep AVM Subtypes}

A solid understanding of the anatomy of deep AVMs is critical for appropriate patient selection and safe surgical resection. The deep core of the cerebrum is where the telencephalon and diencephalon fuse during embryological development. This complex region has been variously described as the insular block, central area, and central core, among other names. Surgically, we find it most informative to categorize AVMs in this territory as insular, basal ganglia, or thalamic.

Insular AVMs are in insular cortex, in the limen insulae, short gyri, and long gyri. They can lie on or extend into insular cortex, but respect the claustrum to spare deeper basal ganglia structures (Figure 3). The insular segments (M2 segments) of the middle cerebral artery lie above or lateral to the AVM, requiring the resection to proceed between these arterial branches. Insular AVMs are included in Sugita's classification of Sylvian fissure AVMs, which he described as deep Sylvian AVMs. ${ }^{16,17}$ Other AVMs in this classification were: 1) the lateral Sylvian fissure AVM based in the temporal lobe and lying along the lateral surface of the fissure; 2) the medial Sylvian fissure AVM based in the frontal lobe and lying along the medial surface of the fissure; and 3) the pure Sylvian fissure AVM not based in a lobe but lying entirely in the fissure around the MCA vessels. These other Sylvian AVMs were excluded from our review.

Basal ganglia AVMs extend deep to insular cortex and into the caudate nucleus, putamen, globus pallidus, and/or anterior limb of the internal capsule (Figure 4). They are also deep to M2 segments, but deeper than insular AVMs and they sit lateral to the posterior limb of the internal capsule. Thalamic AVMs lie in the thalamus and are medial to the posterior limb of internal capsule (Figure 5).

Insular AVMs are supplied by the MCA, either through large branches off the M2 segments, small insular perforators, or lenticulostriate arteries off the M1 segment. Feeding arteries are 
often transit or en passage arteries that continue to supply normal brain in distal territories. Basal ganglia AVMs are supplied by these same 3 types of MCA vessels but also by the AChA. In contrast, thalamic AVMs are supplied from below by thalamoperforating arteries (from the P1 PCA and posterior communicating artery), posterior choroidal arteries (lateral more often than medial), and the AChA. Insular AVMs were the most embolized of the deep AVMs, reflecting their supply by large MCA branches. The small caliber of perforators supplying these AVMs, the difficulty in catheterizing them, and the high risk associated with embolization accounts for a low rate of preoperative embolization (42\%), relative to AVMs in other locations (parafalcine, 74\%; temporal, 67\%; and cerebellar, 55\% ${ }^{18,19}$ ). Transsylvian approaches to insular and basal ganglia AVMs give the neurosurgeon early access to the feeding arteries, whereas transcallosal and transcortical approaches to thalamic AVMs deny access to feeding arteries until late in the dissection.

Venous drainage patterns differed between subtypes, with deep venous drainage in $56 \%$ of insular, $80 \%$ of basal ganglia, and $100 \%$ of thalamic AVMs. Superficial veins drained through lateral subependymal veins and Sylvian veins; deep veins drained medially to thalamostriate and internal cerebral veins, or inferiorly to basal veins of Rosenthal. Arterialized Sylvian veins are good landmarks to basal ganglia and insular AVMs located below insular cortex during transsylvian approaches. Similarly, arterialized ventricular veins are good landmarks to thalamic and basal ganglia AVMs during transcallosal approaches.

\section{Surgical Approaches}

Selection of the optimal surgical approach is critical to successful deep AVM resection, and there are many options (Figure 1). Like with cavernous malformations, ${ }^{20}$ a systematic review of surgical options can help to choose an approach that maximizes exposure of the AVM, directly accesses all margins of the nidus, and minimizes brain transgression and retraction.

Overall, 10 different approaches were used in our experience with deep AVMs (Table 1, Figure 1). To summarize, insular AVMs were exposed with anterior and posterior transsylvian-transinsular approaches. Most basal ganglia AVMs were also exposed with a transsylvian-transinsular approach, which specifically accessed AVMs in the putamen and anterior limb of internal capsule (inferolateral basal ganglia). AVMs in the caudate nucleus (superolateral basal ganglia) were exposed with a contralateral transcallosal approach. AVMs in the globuspallidus (inferomedial basal ganglia) were exposed with the supracarotid-infrafrontal approach. Thalamic AVMs were exposed transcallosally, with either an anterior-ipsilateral approach for AVMs in the superior thalamus, a posterioripsilateral approach for AVMs in the posterior thalamus, or transchoroidally for AVMs in the medial thalamus. In the presence of a sizable hematoma or encephalomalacia connecting the cortical surface with the AVM, transcortical approaches (transfrontal, transtemporal, or transparietal) were used instead of subarachnoid and transventricular routes. In short, the surgeon should exploit direct, nonanatomical corridors to deep AVMs when they already exist and otherwise select anatomical corridors through subarachnoid spaces (Sylvian or interhemispheric fissures) and ventricles that minimize brain transgression. 


\section{Multimodality Therapy}

Considering the deep location of these AVMs in or near critical structures and the technical challenges of this surgery, patient outcomes in this experience were excellent, with no surgical mortality, good outcomes in $81 \%$, and permanent neurological morbidity in only $6 \%$. These results were achieved because the same cautious management attitude exercised in selecting patients for surgery was also exercised during surgery when deciding on extent of resection. AVM resection was complete in 34 patients (71\%) and incomplete in 14 patients (29\%). For comparison, our complete resection rates for AVMs in other locations were significantly higher: $100 \%$ with cerebellar AVMs; $93 \%$ with temporal AVMs; and 93\% with parafalcine AVMs. ${ }^{18,19}$ Incomplete resections relate to surgical selection, but also the AVM's proximity to the internal capsule and respect for the thalamus. These cases are routinely monitored with intraoperative somatosensory and motor evoked potentials and signal changes alert the neurosurgeon that additional dissection or complete resection might be costly. Complete AVM resection is one of two surgical objectives; preservation of neurological function is the other. Our incomplete resection rate reflects a conservative posture that prioritizes neurological outcomes. Complete resection rates tend to be higher for deep AVMs that present with hemorrhage and significant preoperative deficits, as in the literature review by Gross et al. ${ }^{7}$ that reported a $91 \%$ complete resection rate in a surgical cohort with ruptured AVMs in 94\% (Table 5). Although the presence of a hematoma can define a surgical plane and facilitate resection, these AVMs are critically eloquent and extent of resection must be influenced by intraoperative monitoring and the neurosurgeon's intuition regarding the safety of further dissection.

Consequently, deep AVMs require multimodality management more than other AVMs. Radiosurgery was part of the management of nearly one third of our patients, either before or after surgery. Incomplete resection of AVMs led to smaller size, reduced arterial supply, or circumferential dissection except for the deep plane. These surgical changes could possibly facilitate radiosurgical obliteration and stereotactic radiosurgery was therefore recommended for all patients with incompletely resected AVMs. Radiosurgery was specifically targeted to the deep remnant or tip of the AVM adjacent to the internal capsule and thalamus. Although this subgroup of patients is small and our radiosurgical results are unknown at this time, others have reported obliteration rates of $43-81 \%$ for deep AVMs. ${ }^{4-6,21,22}$ This approach adds risk of hemorrhage during the latency period, which has been reported to be $8-15 \% \%^{4-6,21,22}$ and was $7 \%$ in our experience. Still, we prefer conservative surgery with a multimodality approach over aggressive and perhaps curative resection with greater associated morbidity. Of note, thalamic AVMs were least likely to achieve complete resection, with a $46 \%$ complete resection rate compared to $80 \%$ in both the basal ganglia and insular groups. Although we did not observe postoperative hemorrhages in these patients, this lower rate of complete resection has prompted reconsidering our surgical indications for thalamic AVMs.

\section{Limitations}

There are several limitations to this study. First and foremost, this is a retrospective case series that describes our institution's unique practice with deep AVMs. While we summarized general principles for patient selection, unique factors are taken into 
consideration with each patient when deciding management strategy and are difficult to capture in a retrospective review. Such factors include medical comorbidities, patient preferences, and individual biases of members of our multidisciplinary team. Second, the subset of patients with subtotal resections treated subsequently with radiosurgery lacks adequate follow-up, and a conclusive statement regarding the success of this multimodality approach cannot be made. This is an important aspect of deep AVM treatment that we intend to analyze in the future. Third, while we performed multivariate analysis of factors associated with complete resection, we realize that the patient cohort is already highly selected, chosen in part because complete resection was considered possible. Finally, we acknowledge that our relatively high volume of AVMs operated during this study (over 500 patients) may limit the generalizability of these surgical results, and they must be applied within the context of an institution's own unique experience.

\section{Conclusion}

This experience advances the notion that deep AVMs can be operable lesions. Patients were highly selected for small size, hemorrhagic presentation, young age, and compactness, factors embodied in the Spetzler-Martin and Supplementary grading systems. Overall, 10 different approaches were used, exploiting direct, transcortical corridors created by hemorrhage or otherwise maximizing anatomical corridors through subarachnoid spaces (Sylvian or interhemispheric fissures) and ventricles that minimize brain transgression. The same cautious attitude exercised in selecting patients for surgery was also exercised in deciding extent of resection, opting for incomplete resection and radiosurgery more than with other AVMs to prioritize neurological outcomes over curative resection. Microsurgery is best suited to AVMs in the insula and basal ganglia, where parenchymal dissection is more tolerable and rates of complete resection higher than in the thalamus.

\section{Acknowledgments}

This research is funded in part by the National Institutes of Health, R01 NS034949 (Dr. Young) and P01 NS044155 (Center for Cerebrovascular Research).

\section{References}

1. Fleetwood IG, Marcellus ML, Levy RP, Marks MP, Steinberg GK. Deep arteriovenous malformations of the basal ganglia and thalamus: natural history. J Neurosurg. 2003; 98(4):747750. [PubMed: 12691399]

2. Stapf C, Mast H, Sciacca RR, et al. Predictors of hemorrhage in patients with untreated brain arteriovenous malformation. Neurology. 2006; 66(9):1350-1355. [PubMed: 16682666]

3. Sasaki T, Kurita H, Saito I, et al. Arteriovenous malformations in the basal ganglia and thalamus: management and results in 101 cases. J Neurosurg. 1998; 88(2):285-292. [PubMed: 9452237]

4. Pollock BE, Gorman DA, Brown PD. Radiosurgery for arteriovenous malformations of the basal ganglia, thalamus, and brainstem. J Neurosurg. 2004; 100(2):210-214. [PubMed: 15086226]

5. Kiran NAS, Kale SS, Kasliwal MK, et al. Gamma knife radiosurgery for arteriovenous malformations of basal ganglia, thalamus and brainstem--a retrospective study comparing the results with that for AVMs at other intracranial locations. Acta Neurochir (Wien). 2009; 151(12):15751582. [PubMed: 19415175] 
6. Kano H, Kondziolka D, Flickinger JC, et al. Stereotactic radiosurgery for arteriovenous malformations, Part 4: management of basal ganglia and thalamus arteriovenous malformations. $\mathrm{J}$ Neurosurg. 2012; 116(1):33-43. [PubMed: 22077451]

7. Gross BA, Duckworth EAM, Getch CC, Bendok BR, Batjer HH. Challenging traditional beliefs: microsurgery for arteriovenous malformations of the basal ganglia and thalamus. Neurosurgery. 2008; 63(3):393-410. [PubMed: 18812951]

8. Potts MB, Chang EF, Young WL, Lawton MT, For the UCSF Brain AVM Study Project. Transsylvian-Transinsular Approaches to the Insula and Basal Ganglia: Operative Techniques and Results with Vascular Lesions. Neurosurgery. 2011

9. Waldron JS, Lawton MT. The supracarotid-infrafrontal approach: surgical technique and clinical application to cavernous malformations in the anteroinferior Basal Ganglia. Neurosurgery. 2009; 64(3 Suppl):86-95. [PubMed: 19050656]

10. Shucart WA, Stein BM. Transcallosal approach to the anterior ventricular system. Neurosurgery. 1978; 3(3):339-343. [PubMed: 740132]

11. Lawton MT, Golfinos JG, Spetzler RF. The contralateral transcallosal approach: experience with 32 patients. Neurosurgery. 1996; 39(4):729-34. [PubMed: 8880765]

12. Lawton MT, Du R, Tran MN, et al. Effect of presenting hemorrhage on outcome after microsurgical resection of brain arteriovenous malformations. Neurosurgery. 2005; 56(3):485-93. [PubMed: 15730573]

13. Rhoton AL, Yamamoto I, Peace DA. Microsurgery of the third ventricle: Part 2. Operative approaches. Neurosurgery. 1981; 8(3):357-373. [PubMed: 7242884]

14. Lawton MT. Spetzler-Martin Grade III Arteriovenous Malformations: Surgical Results and a Modification of the Grading Scale. Neurosurgery. 2003; 52(4):740-749. [PubMed: 12657169]

15. Lawton MT, Kim H, McCulloch CE, Mikhak B, Young WL. A supplementary grading scale for selecting patients with brain arteriovenous malformations for surgery. Neurosurgery. 2010; 66(4): 702-13. [PubMed: 20190666]

16. Sugita K, Takemae T, Kobayashi S. Sylvian fissure arteriovenous malformations. Neurosurgery. 1987; 21(1):7-14. [PubMed: 3614608]

17. Lawton MT, Lu DC, Young WL, University of California, San Francisco Brain Arteriovenous Malformation Study Project. Sylvian fissure arteriovenous malformations: an application of the Sugita classification to 28 surgical patients. Neurosurgery. 2007; 61(1):29-36. [PubMed: 17621016]

18. Kim YB, Young WL, Lawton MT, UCSF BAVM Study Project. Parafalcine and midline arteriovenous malformations: surgical strategy, techniques, and outcomes. J Neurosurg. 2011; 114(4):984-993. [PubMed: 21250805]

19. Rodríguez-Hernández A, Kim H, Pourmohamad T, Young WL, Lawton MT, University of California, San Francisco Arteriovenous Malformation Study Project. Cerebellar Arteriovenous Malformations: Anatomic Subtypes, Surgical Results, and Increased Predictive Accuracy of the Supplementary Grading System. Neurosurgery. 2012; 71(6):1111-1124. [PubMed: 22986595]

20. Chang EF, Gabriel RA, Potts MB, Berger MS, Lawton MT. Supratentorial cavernous malformations in eloquent and deep locations: surgical approaches and outcomes. Clinical article. J Neurosurg. 2011; 114(3):814-827. [PubMed: 20597603]

21. Nicolato A, Foroni R, Crocco A, et al. Gamma Knife Radiosurgery in the Management of Arteriovenous Malformations of the Basal Ganglia Region of the Brain. Minim Invasive Neurosurg. 2002; 45(4):211-223. [PubMed: 12494356]

22. Nagy G, Major O, Rowe JG, et al. Stereotactic Radiosurgery for Arteriovenous Malformations Located in Deep Critical Regions. Neurosurgery. 2011

23. Shi YQ, Chen XC. Surgical treatment of arteriovenous malformations of the striatothalamocapsular region. J Neurosurg. 1987; 66(3):352-356. [PubMed: 3819829]

24. Solomon RA, Stein BM. Interhemispheric approach for the surgical removal of thalamocaudate arteriovenous malformations. J Neurosurg. 1987; 66(3):345-351. [PubMed: 3819828]

25. U HS, Kerber CW, Todd MM. Multimodality treatment of deep periventricular cerebral arteriovenous malformations. Surg Neurol. 1992; 38(3):192-203. [PubMed: 1440204] 
26. Tew JM, Lewis AI, Reichert KW. Management strategies and surgical techniques for deep-seated supratentorial arteriovenous malformations. Neurosurgery. 1995; 36(6):1065-1072. [PubMed: 7643983]

27. Lawton MT, Hamilton MG, Spetzler RF. Multimodality treatment of deep arteriovenous malformations: thalamus, basal ganglia, and brain stem. Neurosurgery. 1995; 37(1):29-35. [PubMed: 8587687]

28. de Oliveira E, Tedeschi H, Siqueira MG, Ono M, Rhoton AL. Arteriovenous malformations of the basal ganglia region: rationale for surgical management. Acta Neurochir (Wien). 1997; 139(6): 487-506. [PubMed: 9248582]

\section{Abbreviations}

$\begin{array}{ll}\text { AVM } & \text { arteriovenous malformations } \\ \text { MCA } & \text { middle cerebral artery } \\ \text { RS } & \text { Rankin Scale }\end{array}$




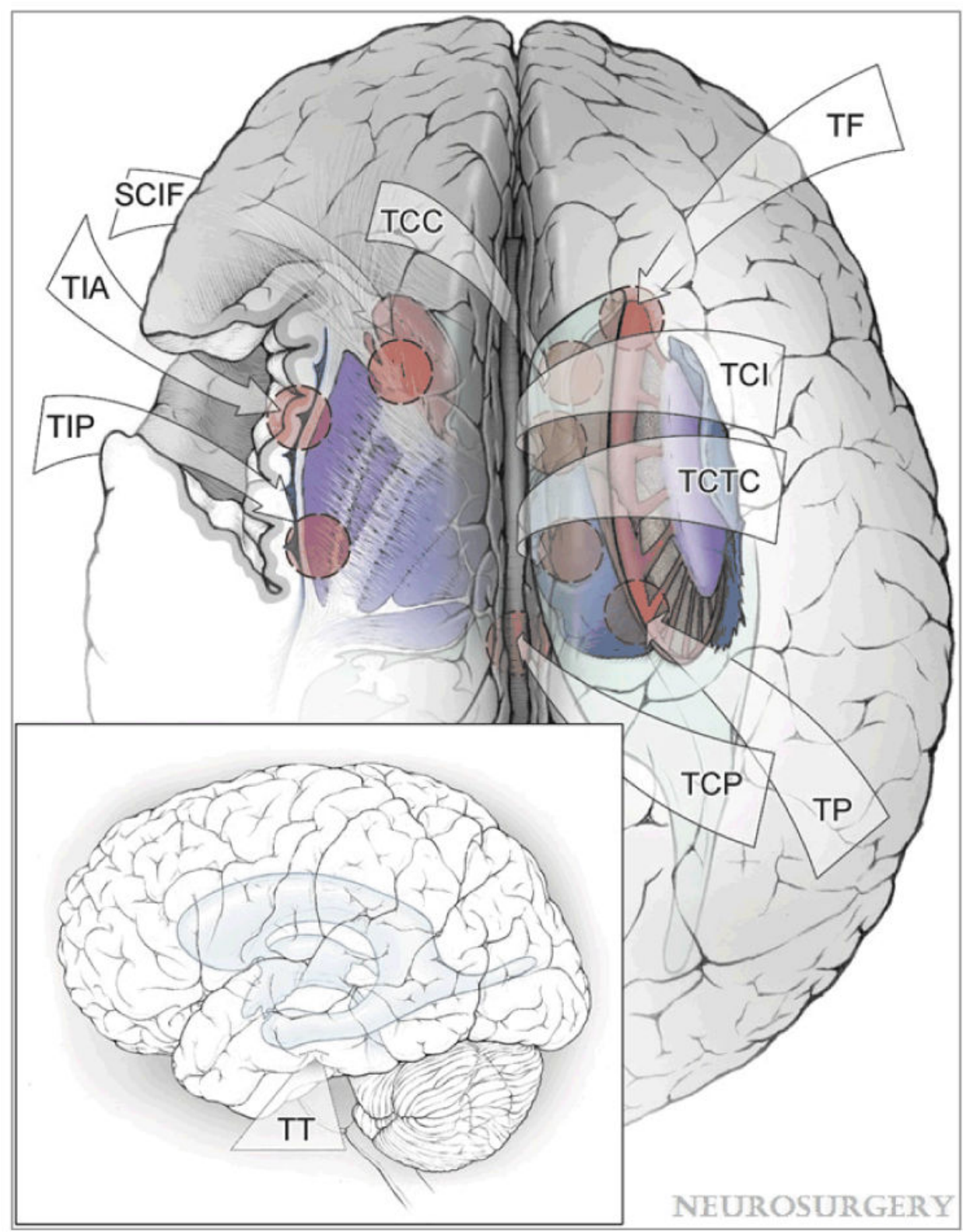

Figure 1.

Overview of surgical approaches to deep AVMs. Transylvian approaches include: anterior transinsular approach (TIA), posterior transinsular approach (TIP), and supracarotidinfrafrontal approach (SCIF). Transcallosal approaches include: ipsilateral transcallosal approach (TCI), contralateral transcallosal approach (TCC), transcallosal-transchoroidal fissure approach (TCTC), and posterior transcallosal approach (TCP). Transcortical approaches include transfrontal (TF), transparietal (TP), and transtemporal (TT) approaches. 


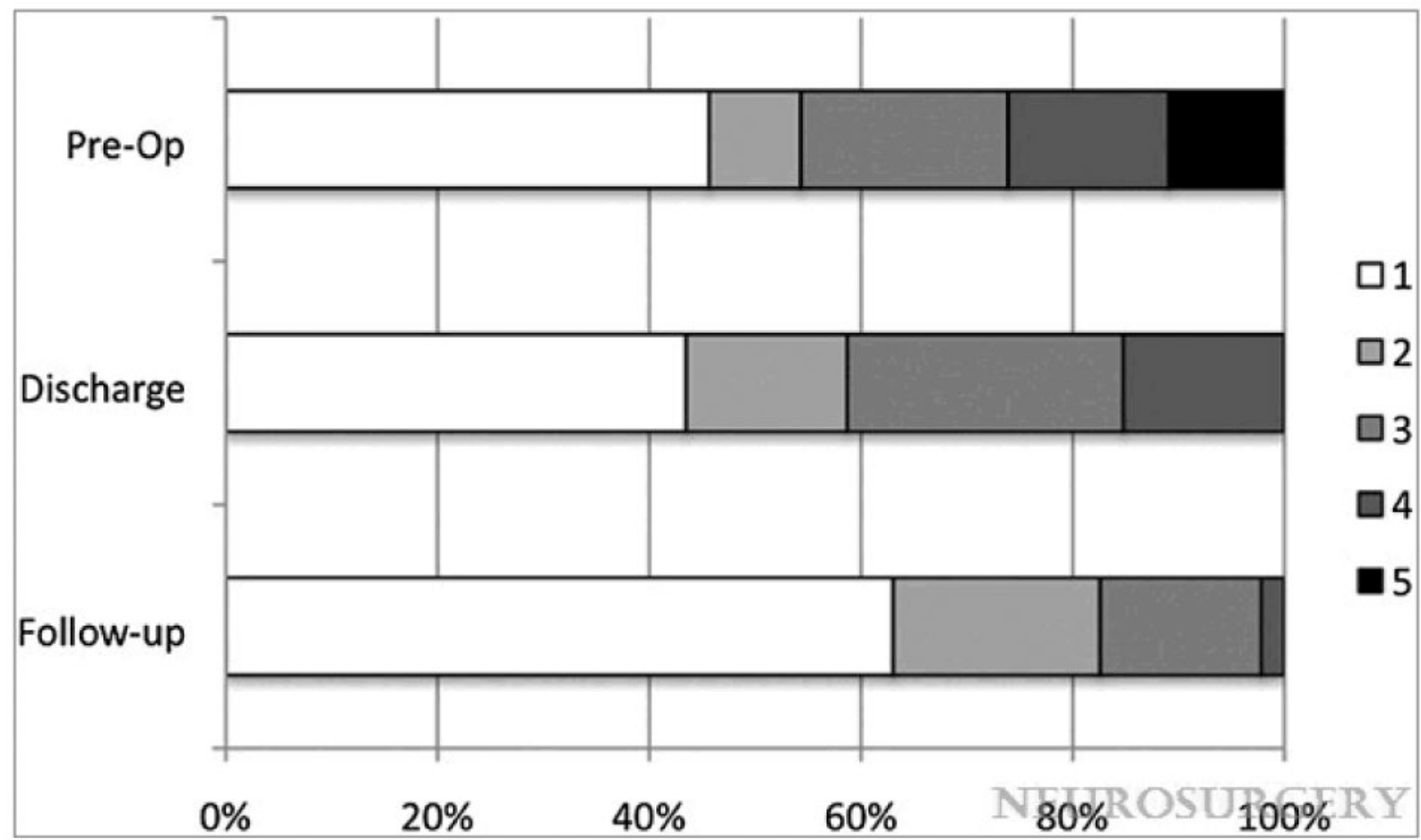

Figure 2.

Summary of neurological condition at presentation and neurological outcomes at early and late follow-up based on the Rankin scale (RS). 
A

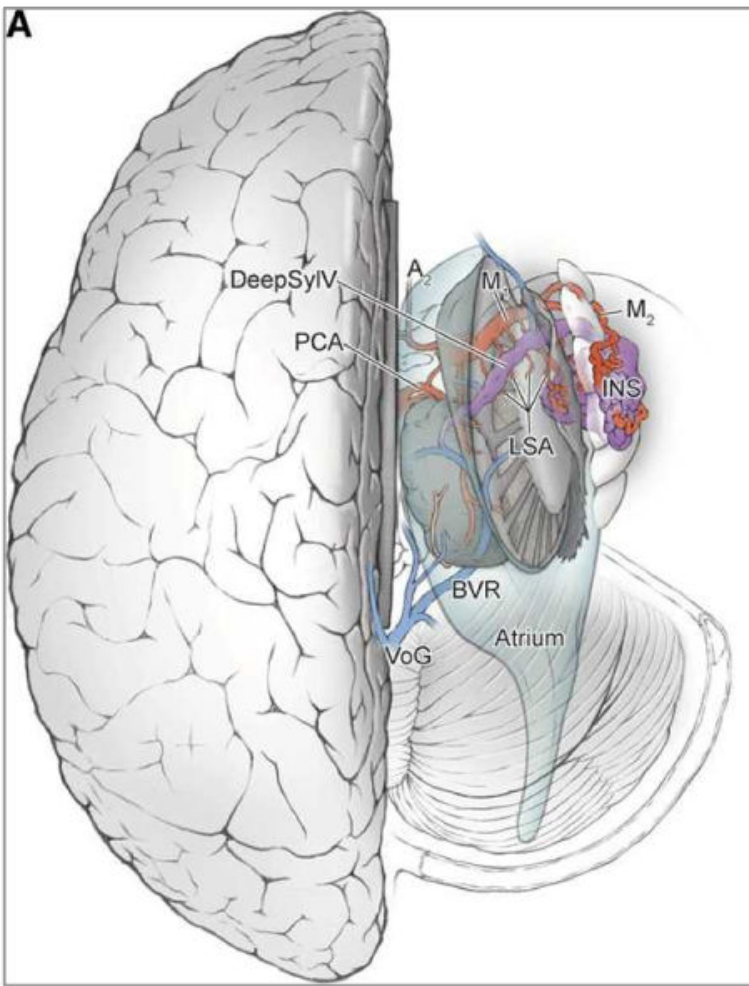

B

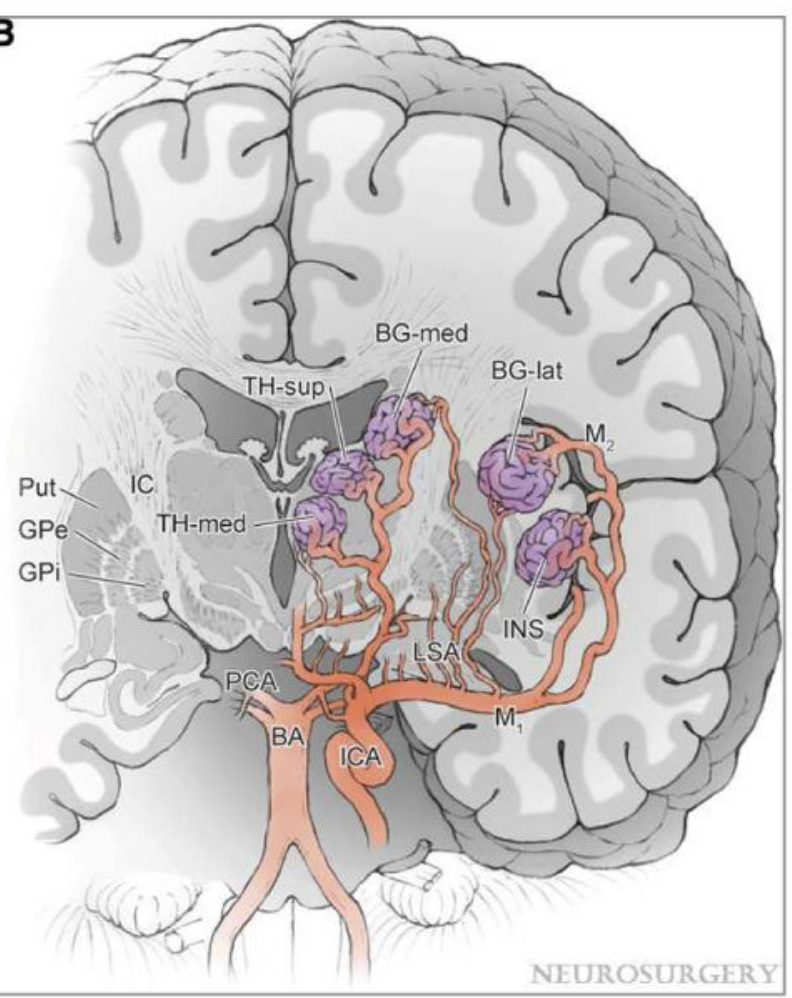

Figure 3.

$\mathrm{A}$, insular arteriovenous malformation (AVM) subtype (INS), as seen in the right insula from above, with the overlying cerebrum removed. B, insular AVM, as seen in the left insula from anterior, in a coronal slice. The coronal transection also shows the other deep AVM subtypes: lateral (BG-lat) and medial (BG-med) basal ganglia AVMs and superior (TH-sup) and medial (TH-med) thalamic AVMs. BA, basilar artery; BVR, basal vein of Rosenthal; DeepSylV, deep sylvian vein; GPe, globus pallidus pars external; GPi, globus pallidus pars internal; IC, internal capsule; ICA, internal carotid artery; LSA, lenticulostriate artery; M1, middle cerebral artery, M1 segment; M2, middle cerebral artery, M2 segment; PCA, posterior cerebral artery; Put, putamen; VoG, vein of Galen. 

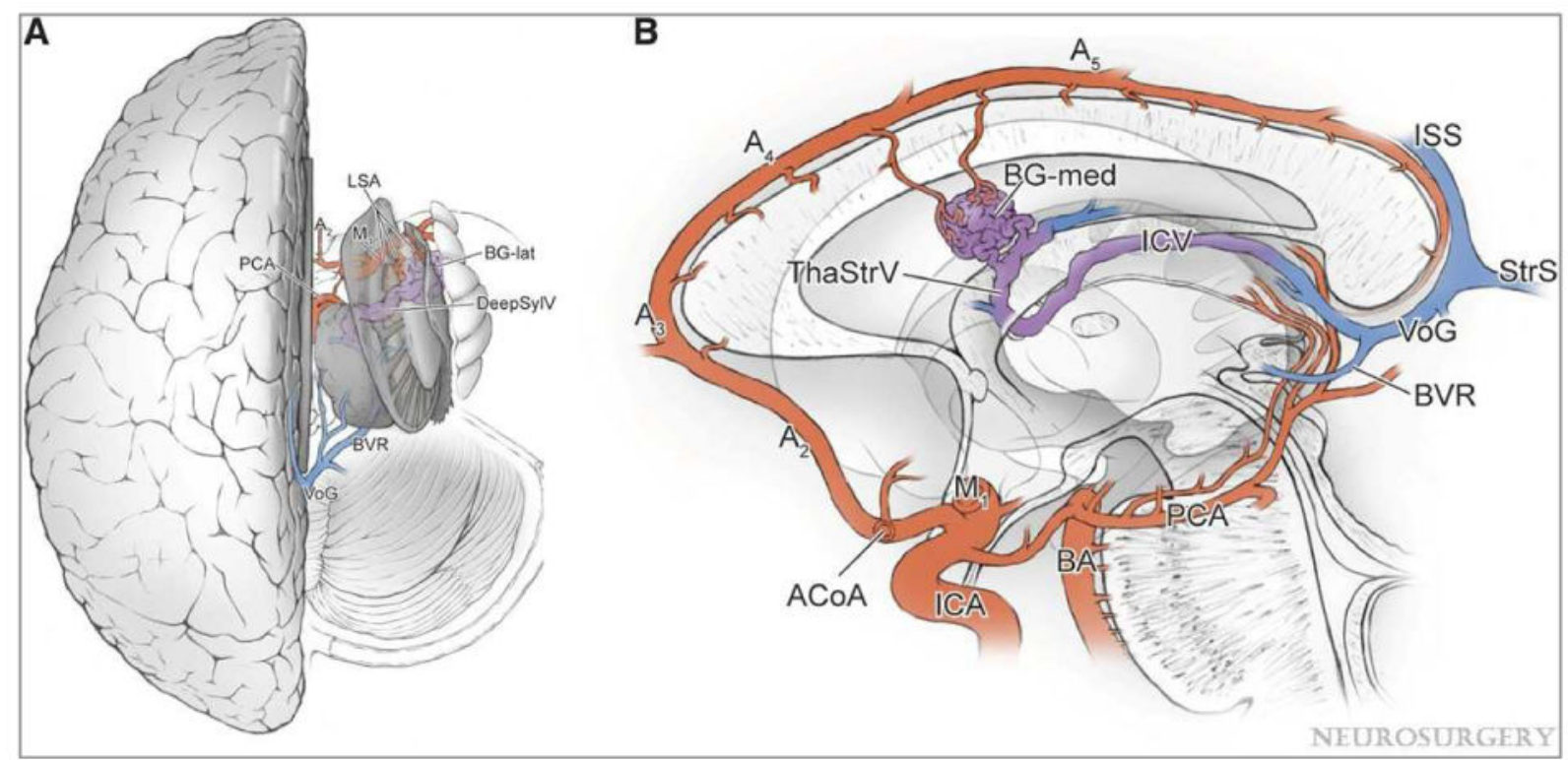

Figure 4.

A, basal ganglia arteriovenous malformation (AVM) subtype, as seen in the right putamen from above, with the overlying cerebrum removed. Basal ganglia AVMs can be located laterally (BG-lat), deep to the insula or (B) medially (BG-med), on or deep to the lateral wall of the lateral ventricle. ACoA, anterior communicating artery; A2, anterior cerebral artery, A2 segment; BA, basilar artery; BVR, basal vein of Rosenthal; DeepSylV, deep sylvian vein; GPe, globus pallidus pars external; GPi, globus pallidus pars internal; IC, internal capsule; ICA, internal carotid artery; ISS, inferior sagittal sinus; LSA, lenticulostriate artery; M1, middle cerebral artery, M1 segment; PCA, posterior cerebral artery; StrS, straight sinus; ThalStrV, thalamostriate vein; VoG, vein of Galen. 


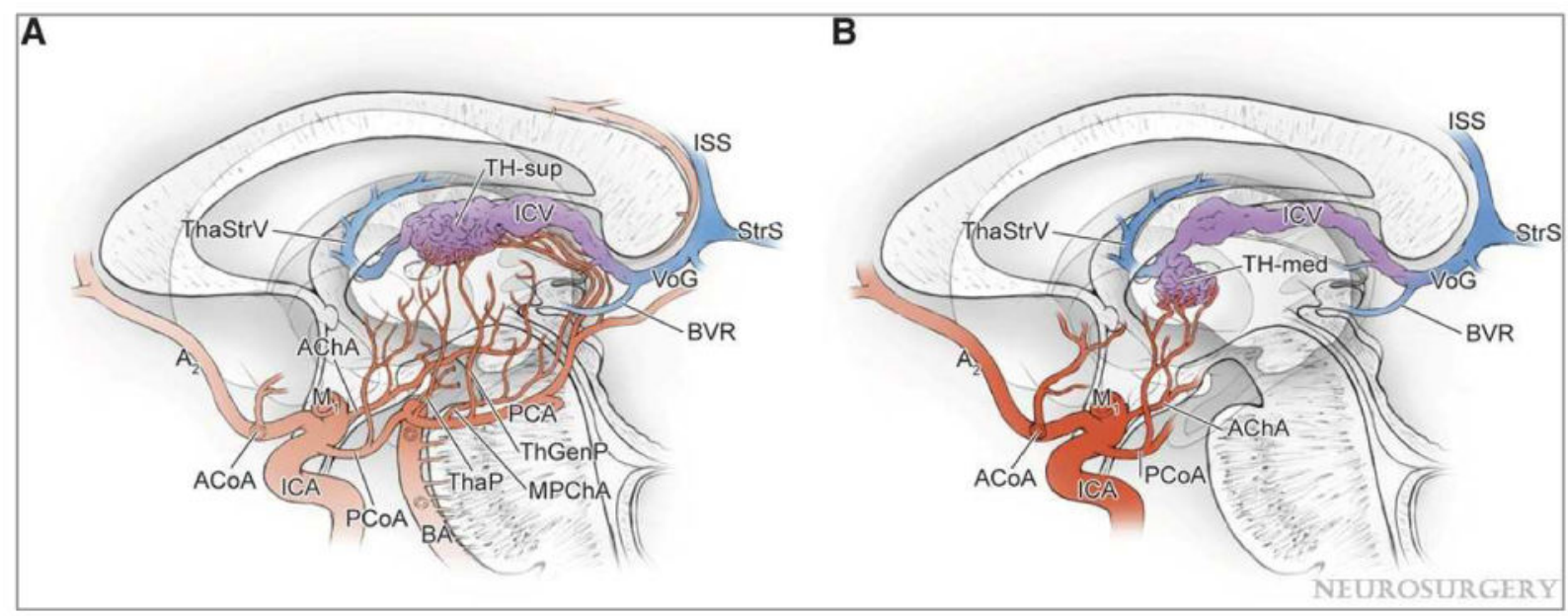

Figure 5.

A, superior thalamic (TH-sup) AVM Study Project arteriovenous malformation (AVM), as seen in the sagittal midline cross section. Superior thalamic AVMs lie on the upper surface of the thalamus that forms the floor of the lateral ventricle. B, medial thalamic AVMs (THmed) lie on the medial surface of the thalamus that forms the lateral wall of the third ventricle. AChA, anterior choroidal artery; ACoA, anterior communicating artery; A2, anterior cerebral artery, A2 segment; A3, anterior cerebral artery, A3 segment; A4, anterior cerebral artery, A4 segment; BA, basilar artery; BVR, basal vein of Rosenthal; DeepSylV, deep sylvian vein; GPe, globus pallidus pars external; GPi, globus pallidus pars internal; IC, internal capsule; ICA, internal carotid artery; ISS, inferior sagittal sinus; LSA, lenticulostriate artery; MPChA, medial posterior choroidal artery; M1, middle cerebral artery, M1 segment; M2, middle cerebral artery, M2 segment; PCA, posterior cerebral artery; Put, putamen; StrS, straight sinus; ThalStrV, thalamostriate vein; ThaP, thalamoperforators; ThGenP, thalamogeniculate perforators; VoG, vein of Galen. 


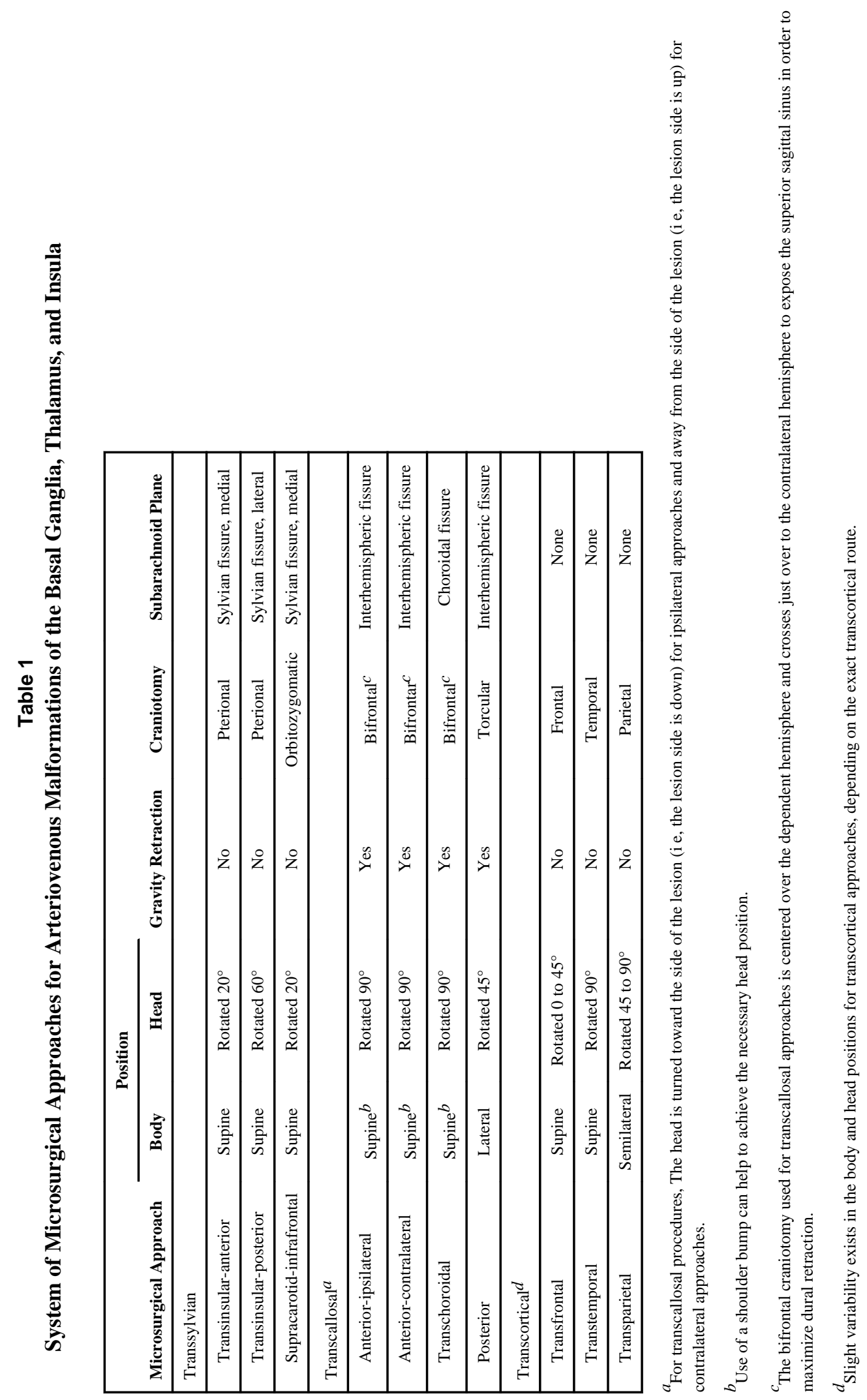

Neurosurgery. Author manuscript; available in PMC 2014 September 01. 


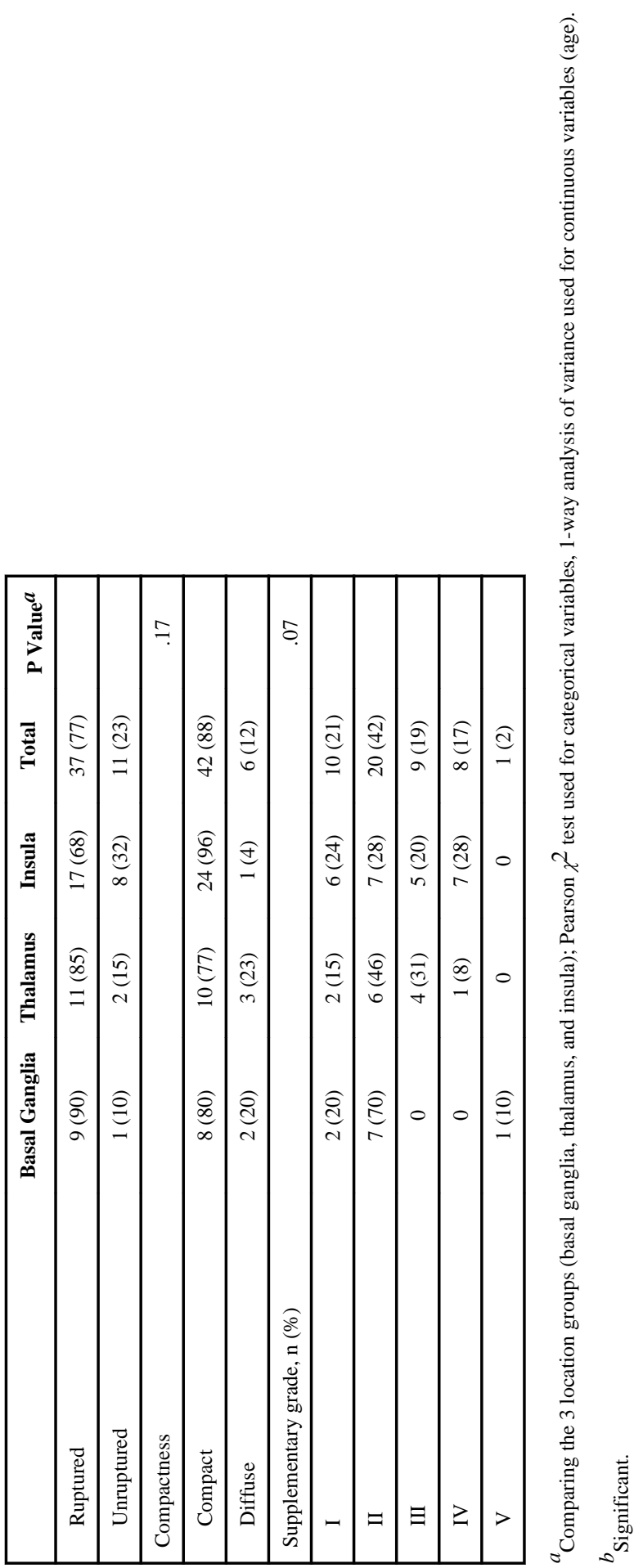

Neurosurgery. Author manuscript; available in PMC 2014 September 01. 


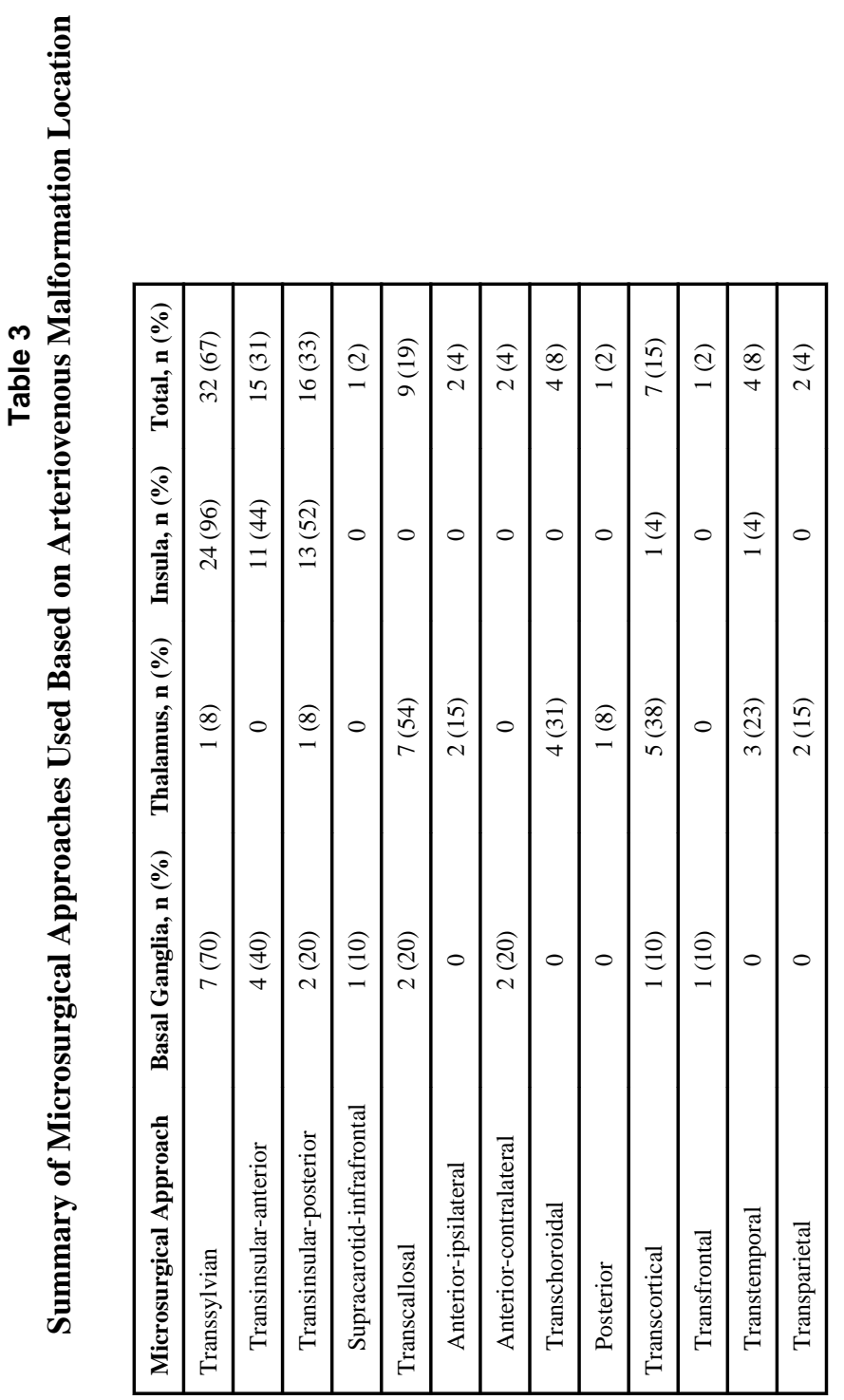

Neurosurgery. Author manuscript; available in PMC 2014 September 01. 


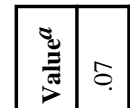

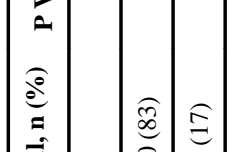

氶

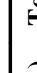

อ

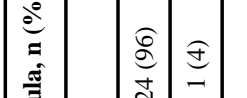

4
0
$=$
$=$

它

so

产

(ะ)

至

बิa

恿

兽

릉

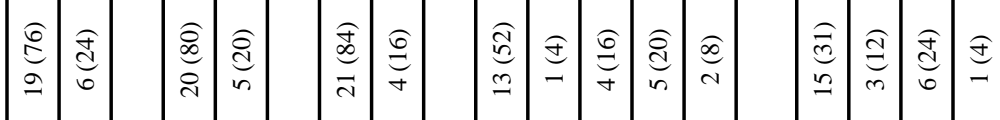

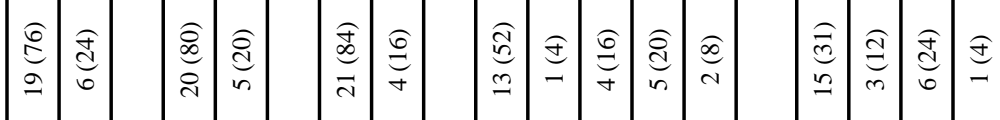

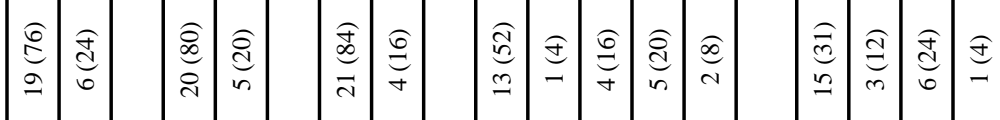

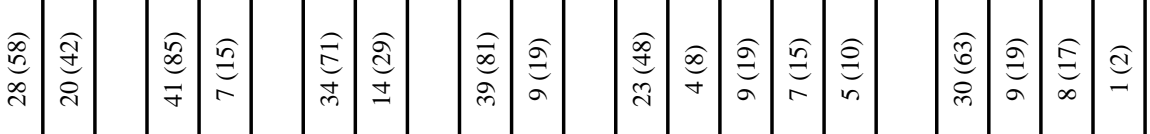

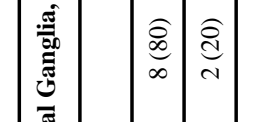

焉 


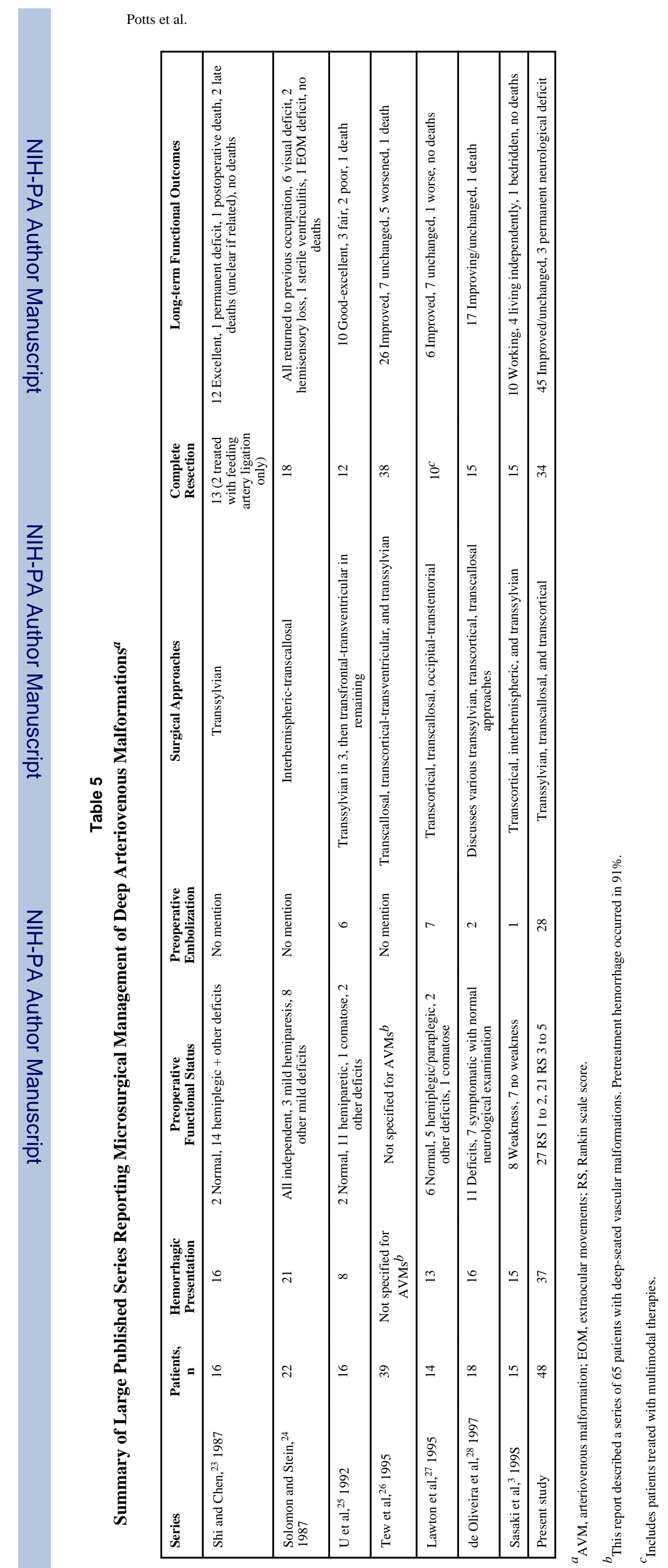

Page 25 